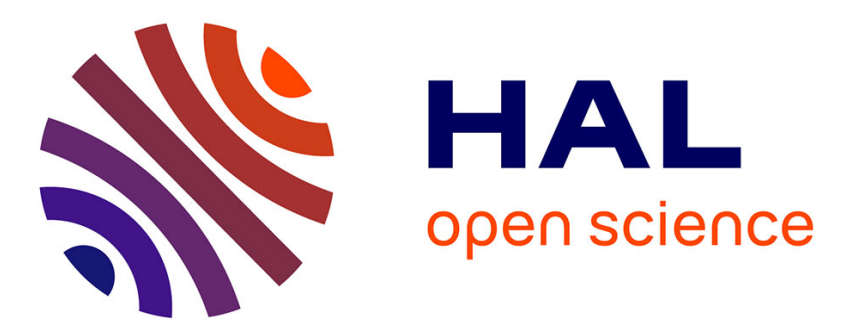

\title{
Fragments du jeu académique postcolonial (à propos d'un collectif, l'Association pour la connaissance de l'histoire de l'Afrique contemporaine, ACHAC) Vincent Chambarlhac
}

\section{- To cite this version:}

Vincent Chambarlhac. Fragments du jeu académique postcolonial (à propos d'un collectif, l'Association pour la connaissance de l'histoire de l'Afrique contemporaine, ACHAC). Histoire@Politique: revue du Centre d'histoire de Sciences Po, 2010, 12 (12), pp.1-12. 10.3917/hp.012.0012 . halshs-00555774

\section{HAL Id: halshs-00555774 \\ https://shs.hal.science/halshs-00555774}

Submitted on 25 Oct 2017

HAL is a multi-disciplinary open access archive for the deposit and dissemination of scientific research documents, whether they are published or not. The documents may come from teaching and research institutions in France or abroad, or from public or private research centers.
L'archive ouverte pluridisciplinaire HAL, est destinée au dépôt et à la diffusion de documents scientifiques de niveau recherche, publiés ou non, émanant des établissements d'enseignement et de recherche français ou étrangers, des laboratoires publics ou privés. 


\section{FRAGMENTS DU JEU ACADÉMIQUE POSTCOLONIAL (À PROPOS D'UN COLLECTIF, L'ASSOCIATION POUR LA CONNAISSANCE DE L'HISTOIRE DE L'AFRIQUE CONTEMPORAINE, ACHAC)} Vincent Chambarlhac

Centre d'histoire de Sciences Po | «Histoire@Politique »

2010/3 n 12 | pages 12 à 12

\section{Article disponible en ligne à l'adresse :}

https://www.cairn.info/revue-histoire-politique-2010-3-page-12.htm

\section{Pour citer cet article :}

Vincent Chambarlhac, "Fragments du jeu académique postcolonial (à propos d'un collectif, l'Association pour la connaissance de l'histoire de l'Afrique contemporaine, ACHAC) », Histoire@Politique 2010/3 (n 12), p. 12-12.

DOI 10.3917/hp.012.0012

Distribution électronique Cairn.info pour Centre d'histoire de Sciences Po.

(C) Centre d'histoire de Sciences Po. Tous droits réservés pour tous pays.

La reproduction ou représentation de cet article, notamment par photocopie, n'est autorisée que dans les limites des conditions générales d'utilisation du site ou, le cas échéant, des conditions générales de la licence souscrite par votre établissement. Toute autre reproduction ou représentation, en tout ou partie, sous quelque forme et de quelque manière que ce soit, est interdite sauf accord préalable et écrit de l'éditeur, en dehors des cas prévus par la législation en vigueur en France. Il est précisé que son stockage dans une base de données est également interdit. 
Vincent Chambarlhac, «Fragments du jeu académique postcolonial (à propos d'un collectif, l'Association pour la connaissance de l'histoire de l'Afrique contemporaine, ACHAC) 》, Histoire@Politique. Politique, culture, société, $N^{\circ} 12$, septembre-décembre 2010, www.histoirepolitique.fr

\title{
Fragments du jeu académique postcolonial (à propos d'un collectif, l'Association pour la connaissance de l'histoire de l'Afrique contemporaine, ACHAC)
}

\author{
Vincent Chambarlhac
}

Pour emblématique qu'il soit, le débat sur l'article 4 de la loi du 23 février 2005 vaut palimpseste, effaçant - parce que le réécrivant - le texte des polémiques intellectuelles qui contribuèrent à la structuration de la controverse coloniale. Partant, il ne s'agit pas pour autant de restituer un sens caché à ce débat ${ }^{1}$, mais plutôt d'évoquer à grands traits un faisceau serré de pratiques universitaires, éditoriales et militantes qui concourent à cette structuration dont le nœud gordien serait le lien tissé entre le passé colonial et le présent républicain. Soit l'irruption des postcolonial studies dans le champ académique. Si l'essentiel de mon propos vise à restituer à une part des acteurs de ce débat leur rôle singulier, le politique - dans sa version parlementaire et partidaire - demeure en hors-champ de l'analyse. Dans la genèse intellectuelle du débat, ces jeux d'acteurs se saisissent à mes yeux dans l'horizon du postcolonialisme comme enjeu conceptuel simultanément universitaire et militant, à l'orée des années 1990 dans le monde anglo-saxon ${ }^{2}$, aujourd'hui en France. Embrasser l'ensemble du champ français du postcolonialisme outrepasse évidemment le cadre de cet article; il se restreint à la part active prise dans le développement de ce champ par Nicolas Bancel, Pascal Blanchard et plus largement le collectif qu'est l'Association pour la connaissance de l'histoire de l'Afrique contemporaine (ACHAC) dans son lien à l'histoire contemporaine. Dans l'historiographie française, leurs publications, et les stratégies qui les animent, constituent à mes yeux une part de la trame des débats en cours sur le postcolonial.

L'hypothèse d'un transfert culturel ouvre l'analyse ; elle n'est pas inédite, reprend un argument souvent brandi dans les débats. Cette hypothèse débouche sur la saisie de deux logiques complémentaires. L'une se situe aux confins des pratiques universitaires et médiatiques; elle désigne autant un projet qu'une manière de se situer dans le champ de l'histoire universitaire. La seconde désigne l'appropriation politique de la problématique postcoloniale dans l'espace public, et postule que celleci participe de l'inscription du postcolonial dans le jeu académique. Toutes deux

\footnotetext{
${ }^{1}$ Romain Bertrand, Mémoires d’empire. La controverse autour du « fait colonial », Clamecy, Editions du Croquant « Savoir agir », 2006.

2 Neil Lazarus, Penser le postcolonial, une introduction critique, Paris, Editions Amsterdam, 2006.
} 
Vincent Chambarlhac, «Fragments du jeu académique postcolonial (à propos d'un collectif, l'Association pour la connaissance de l'histoire de l'Afrique contemporaine, ACHAC) », Histoire@Politique. Politique, culture, société, $N^{\circ} 12$, septembre-décembre 2010, www.histoirepolitique.fr

signalent des lieux et des passeurs par quoi et par qui les débats adviennent, se structurent et s'arriment au champ historique dans la récurrence des polémiques.

\section{L'hypothèse d'un transfert culturel}

Sur le fond, ce qui se joue dans le débat sur le fait colonial tient à ce que l'histoire de ce passé fait aux universaux républicains. Cette position travaille finalement peu le monde colonial: seule importe la métropole. Lié au présentisme des enjeux mémoriels, ce questionnement de la République à partir du fait colonial convoque les apports des postcolonial studies anglo-saxonnes. Ce mouvement, qui commence à partir du début des années 1990, se déploie à partir de la culture comme objet. Significativement, l'une des premières manifestations de cette irruption des problématiques des postcolonial et cultural studies dans l'historiographie française est la longue recension que Christophe Prochasson donne de l'ouvrage d'Hermann Lebovics pour les Annales HSS ${ }^{3}$ - ce livre est finalement traduit chez Belin en 1995. Pour Christophe Prochasson, «le retour à une histoire politique passe par la publication » de tels ouvrages ${ }^{4}$. C'est dans la conjoncture du renouvellement de l'histoire politique par l'histoire culturelle que se déploient l'appel aux travaux anglosaxons et, dans le cas présent, la question du postcolonialisme. La situation peut se lire dans la problématique de l'appel au profane : en recourant aux travaux anglosaxons, une partie des historiens français se distingue dans la reconfiguration en cours du champ historiographique par l'histoire culturelle ${ }^{5}$. Dans sa recension, Christophe Prochasson salue l'éclectisme et le brio des références sociologiques et historiographiques d'Hermann Lebovics. Ce jeu de miroir, propre au fonctionnement réticulaire du champ historique, indique pour partie le bénéfice d'un appel au cultural studies: la légitimation, par le pragmatisme anglo-saxon, de l'empirisme présidant à l'histoire culturelle. Cette légitimation décloisonne le champ universitaire dans un moment marqué par le sentiment d'une crise de l'histoire. La publication française de La Vraie France ouvre ainsi la voie à toute une série de travaux français engagés dans les problématiques d'Hermann Lebovics ${ }^{6}$.

L'appel à l'historiographie anglo-saxonne participe de la modification en profondeur de l'économie de l'histoire politique puisqu'elle décentre le regard à partir des objets — c'est là la réussite d'une histoire culturelle du politique -, à partir également des concepts et des échelles mêmes de leur application. On peut ainsi risquer l’hypothèse,

\footnotetext{
3 Hermann Lebovics, La Vraie France. Les enjeux de l'identité culturelle (1900-1945), Paris, Belin, 1995. ${ }^{4}$ Christophe Prochasson, Annales HSS, vol. 49, n 1, 1994, p. 161-163.

5 Philippe Poirrier (dir.), L'histoire culturelle, un «tournant mondial dans l'historiographie», Dijon, EUD, 2008.

6 Par exemple : Pascal Blanchard et alii, Culture coloniale. La France conquise par son Empire (18711931), Paris. Autrement « Mémoires », 2003. Egalement, Pascal Blanchard et alii, Culture impériale (1931-1961). Les colonies au cour de la République, Paris, Autrement, coll. « Mémoires », 2004. Carole Raynaud Paligot, La République raciale, 1830-1930, Paris, Presses universitaires de France, 2006.
} 
Vincent Chambarlhac, «Fragments du jeu académique postcolonial (à propos d'un collectif, l'Association pour la connaissance de l'histoire de l'Afrique contemporaine, ACHAC) », Histoire@Politique. Politique, culture, société, $N^{\circ} 12$, septembre-décembre 2010, www.histoirepolitique.fr

à propos de la question coloniale, d'un transfert culturel paradoxal. Les travaux d’Hermann Lebovics, et de manière plus ample l'ensemble des cultural studies, se sont nourris sur les campus anglo-saxons de la French Theory ${ }^{7}$, qui les charpente pour partie. Son retour sur la scène intellectuelle française, après finalement son appropriation restreinte durant la décennie 1970, relève d'une redécouverte. Pour autant, les effets de ce transfert culturel ne se limitent pas au retour des problématiques de Michel Foucault, Roland Barthes, Pierre Bourdieu. L'irruption des thématiques propres aux postcolonial studies bouscule en partie l'histoire du fait colonial naguère pratiquée à partir de l'histoire politique et de l'histoire sociale. Déplaçant sur le sol métropolitain la prise en compte du fait colonial, elle fragilise l'équilibre des découpages jusque-là dominants où l'histoire coloniale occupait un secteur historiographique marginal ${ }^{8}$. Cet appel au profane présuppose l'hybridation des synthèses prévalant, multiplie les controverses possibles puisqu'elle postule dans la trame du national (réduite spatialement au métropolitain) la part du colonial, soit l'argument emblématique des racines coloniales de la République au sein de l'histoire politique ${ }^{9}$. Implicitement, l'argumentation se structure dans l'horizon du concept de discrimination positive né sur le campus anglo-saxons ${ }^{10}$. Cette modification est d'autant plus importante qu'elle touche à l'un des pôles de la profession d'historien en France, son rapport à l'identité nationale, qu'il en soit successivement l'un des producteurs (fondation républicaine de la discipline), son praticien (entreprise des Lieux de mémoire par exemple) ou l'expert convoqué à son chevet. C'est à ce point que l'analyse en termes de transfert culturel fait sens, puisque dans la dynamique des échanges elle suppose la part essentiel du jeu des individus.

Ici, le rôle de l'ACHAC et des publications qui lui sont liées paraitt déterminant. Son action se déploie à la charnière de deux logiques. L'une est militante et universitaire ; éditoriale, la seconde se marque davantage à partir de 2003 de signes propres au spectaculaire. Cumulées, ces logiques concourent simultanément à la légitimation universitaire d'une part des travaux du collectif au titre du postcolonialisme, et à l'évidence prescriptive de ces thématiques dans l'espace public. Dans son rapport à la société, l'historien paraît ici thaumaturge puisque légitimant scientifiquement les nécessités d'une politique de la reconnaissance ; dans son rapport à l'historiographie, l’historien paraît là challenger. La possibilité des polémiques et l'impossibilité des controverses naissent de ce positionnement singulier ${ }^{11}$.

\footnotetext{
7 Francois Cusset, French Theory. Foucault, Derrida, Deleuze et Cie, et les mutations de la vie intellectuelle aux Etats-Unis, Paris, La Découverte, 2003.

8 Alice Conklin, «Boundaries unbound: teaching French History as Colonial History and Colonial History as French History », French Historical Studies, $n^{\circ} 23$, 2000. Sophie Dulucq, Colette Zytnicki, «Penser le passé colonial français. Entre perspectives historiographiques et résurgences des mémoires », Vingtième siècle. Revue d'histoire, n 86, 2005.

9 Nicolas Bancel, Pascal Blanchard, Françoise Vergès, La République coloniale. Essai sur une utopie, Paris, Albin Michel, 2003.

10 « La discrimination positive », Pouvoirs, n 111, 2004. Gwénaëlle Calvès, La discrimination positive, Paris, PUF, 2004.

${ }^{11}$ La controverse suppose la symétrie des parties en présence; par leur situation à la charnière du militant et du scientifique ces challengers sont toujours lus de manière asymétrique : ils ne sont pas des
} 
Vincent Chambarlhac, «Fragments du jeu académique postcolonial (à propos d'un collectif, l'Association pour la connaissance de l'histoire de l'Afrique contemporaine, ACHAC) », Histoire@Politique. Politique, culture, société, $N^{\circ} 12$, septembre-décembre 2010, www.histoirepolitique.fr

\section{Une trajectoire historiographique}

L'ACHAC participe depuis 1989 d'une manière souvent déterminante à la promotion du concept de culture coloniale ${ }^{12}$. La structure de l'association articule le monde de la recherche (Bancel, Blanchard, et alii), des écrivains (Daeninckx), des cinéastes et des artistes militants, et reproduit en partie la morphologie anglo-saxonne des postcolonial-studies ${ }^{13}$. Son action repose sur des supports variés: l'exposition, le livre, l'article, le documentaire vidéo, la radio... A l'ACHAC, on peut adjoindre, au moins pour son savoir-faire, l'agence les Bâtisseurs de mémoire créée par Pascal Blanchard qui considère l'histoire comme un vecteur de communication au service des entreprises ${ }^{14}$. Les travaux de l'ACHAC ont graduellement balisé l'irruption des thématiques postcoloniales. Ils se déportent progressivement de l'Afrique à la métropole, interpellant alors la République par le biais de la question coloniale.

Trois étapes marquent ce processus.

Dans une première phase, les travaux de l'ACHAC procèdent de la mission fondatrice de l'association, organisant autour de la Bibliothèque de documentation internationale contemporaine (BDIC) et de ses fonds des expositions sur l'Afrique coloniale $^{15}$. Le choix d'une entrée par la culture coloniale implique l'étude des représentations métropolitaines sur le fait colonial. Le catalogue d'exposition prend ainsi comme cible la propagande coloniale, mais aussi dans le sillage d'Edward Saïd, le regard des Occidentaux sur l'Orient ${ }^{16}$, et ce jusqu'en 1962. L'empire colonial français fait également en 1997 l'objet d'une exposition ${ }^{17}$.

A partir de ce capital, une inflexion décisive se dessine avec la publication d'un ouvrage collectif consacré aux zoos humains ${ }^{18}$. Ici, la translation spatiale s'achève. La métropole seule importe comme cadre géographique des représentations coloniales, et la séquence chronologique embrassée par le titre (De la Vénus hottentote aux reality shows) signifie la pertinence d'une grille de lecture postcoloniale. Ce travail sur les zoos humains fut préparé par la publication d'un ouvrage portant sur la continuité de l'indigène à l'immigré ${ }^{19}$. Les travaux d'Abdelmalek Sayad innervent

pairs puisqu'ils ont une place à construire. Le registre de la polémique convient donc davantage pour se saisir des débats sur le postcolonialisme.

12 Philippe Poirrier, Les enjeux de l'histoire culturelle, Paris, Seuil « Points : L'histoire en débat », 2004.

13 Neil Lazarus, Penser le postcolonial, une introduction critique, op. cit.

14 Romain Bertrand, Mémoires d'empire, op. cit., p. 129. Le site des Bâtisseurs de mémoire: www.lesbdm.com

15 ACHAC, Images et colonies (1880-1962). Iconographie et propagande coloniale sur l'Afrique française de 1880 à 1962. Paris, BDIC/ ACHAC, 1993.

16 Edward Saïd, L'orientalisme, Paris, Seuil, 1978.

17 Nicolas Bancel et alii, Images d'empire, Paris, La Documentation française/ La Martinière, 1997.

18 Nicolas Bancel et alii, Zoos humains. De la Vénus hottentote aux reality shows, Paris, La Découverte, 2002.

19 Nicolas Bancel et alii, Del'indigène à l'immigré, Paris, Gallimard, coll. « Découverte », 1998. 
Vincent Chambarlhac, «Fragments du jeu académique postcolonial (à propos d'un collectif, l'Association pour la connaissance de l'histoire de l'Afrique contemporaine, ACHAC) », Histoire@Politique. Politique, culture, société, $N^{\circ} 12$, septembre-décembre 2010, www.histoirepolitique.fr

cette problématique. L'essentiel dans cette translation tient à sa dimension anthropologique où les représentations du corps, ses usages, deviennent le lieu déterminant de l'analyse. La problématique se noue à la discrimination positive, où le corps vaut marqueur social ; elle établit également un pont avec la question de l'esclavage. Au cours de cette seconde étape, l'approche s'institutionnalise scientifiquement par la création du groupe de recherche GDR CNRS 2332 «Anthropologie des représentations du corps » créé en janvier 2001 dans lequel entre l'agence des Bâtisseurs de mémoire, représentée par Pascal Blanchard, et Eric Deroo $^{20}$. Au cours de cette seconde étape, les problématiques employées se resserrent, à partir du concept de culture coloniale, sur la question du rapport à la métropole en termes de représentations. Les objections de Claude Liauzu à ces travaux désignent cette réduction du fait colonial au seul registre des représentations métropolitaines ${ }^{21}$. A ce stade également, l'argument de la culture coloniale s'entend dans la configuration plus ample du succès d'une histoire des représentations dans le champ historique où Sylvain Venayre décèle la fin de la soumission du monde mental au social ${ }^{22}$. Le propos de Claude Liauzu procède en partie du refus de cette fin, comme d'une historiographie aux paradigmes érigés avant le tournant culturaliste.

La troisième étape voit la systématisation de cette entrée sur le fait colonial maintenant placé au cœeur du récit national républicain ${ }^{23}$. L'argument d'une République coloniale publié sous forme d'essai prolonge la trilogie des volumes de la collection Autrement sur la culture coloniale. La publication en 2005 de l'ouvrage consacré à la fracture coloniale ${ }^{24}$ clôt - momentanément - cette étape. Avec ce dernier opus, la grille postcoloniale se donne comme une clé d'explication possible de la question sociale contemporaine. Cinq ans plus tard, le volume Ruptures postcoloniales. Les nouveaux visages de la société française ${ }^{25}$ procède du même mouvement. Par effet retour, ces ouvrages valident ce qui se donnait auparavant sous la forme de l'hypothèse d'une République coloniale, campant ainsi ce collectif d'auteur en challenger d'une réécriture de l'histoire républicaine qui implique l'obsolescence des propositions de Serge Berstein sur le modèle républicain ${ }^{26}$, comme de l'entreprise des Lieux de mémoire dirigée par Pierre Nora. Ici, la révision historique proposée déborde le seul secteur des études sur le monde colonial pour tenter de susciter la controverse scientifique sur les interprétations dominantes de l'histoire politique contemporaine. Cette relecture de l'histoire politique au miroir du colonial emprunte nombre de ses arguments au questionnement des colonial studies anglo-saxonnes comme en témoignent les entrées de Ruptures postcoloniales. Les

\footnotetext{
20 http:// www.anthropologie-biologique.cnrs.fr/ recherche/ consultéle 10/ 04/2007.

${ }^{21}$ Claude Liauzu et alii, Colonisation : droit d'inventaire, Paris, Armand Colin, 2004.

22 Sylvain Venayre, «L'invention de l'invention. L'histoire des représentations en France depuis 1980 », dans Laurent Martin, Sylvain Venayre, L'histoire culturelle du contemporain, Paris, Nouveau Monde éditions, 2005, p. 35.

23 Nicolas Bancel, Pascal Blanchard, Françoise Vergès, La République coloniale. Essai sur une utopie, Paris, Albin Michel, 2003.

24 Nicolas Bancel et alii, La fracture coloniale, Paris, La Découverte, « Cahiers libres », 2005.

25 Nicolas Bancel et alii, Ruptures postcoloniales, Paris, La Découverte, 2010.

${ }^{26}$ Serge Berstein et Odile Rudelle, Le modèle républicain, Paris, PUF, 1992.
} 
Vincent Chambarlhac, «Fragments du jeu académique postcolonial (à propos d'un collectif, l'Association pour la connaissance de l'histoire de l'Afrique contemporaine, ACHAC) », Histoire@Politique. Politique, culture, société, $N^{\circ} 12$, septembre-décembre 2010, www.histoirepolitique.fr

racines intellectuelles d'un collectif s'affirment face à la polémique dans le champ scientifique ouverte par l'irruption de ce postcolonialisme académique ${ }^{27}$.

Ainsi ramassée, cette trajectoire historiographique nouée autour des publications de Nicolas Bancel et de Pascal Blanchard montre comment ceux-ci peuvent apparaître comme des passeurs dans le cadre d'un transfert culturel des problématiques postcoloniales. Peu à peu, autour de leurs publications, s'ébauche un système éditorial qui construit progressivement leur position dans le champ universitaire à partir d'une critique sans cesse plus resserrée des représentations républicaines. En ce sens, leurs travaux s'apparentent à l'émergence d'une nouvelle génération de chercheurs légitimant ses nouveaux champs de recherches par l'appel aux cultural studies. Les controverses universitaires suscitées par ces travaux construisent également, par leur médiatisation, la réputation de ces chercheurs. Cette stratégie part des marges de l'institution universitaire (l'ACHAC, l'agence les Bâtisseurs de mémoire), elle trouve des points d'appui dans le monde universitaire anglo-saxon ${ }^{28}$ et se nourrit de chantiers proches - tel celui de l'esclavage - pour proposer in fine une autre écriture du récit républicain. Ce système éditorial ne sert pas seulement cette position historiographique. La stratégie qui anime son progressif développement structure pour partie la réception des problématiques postcoloniales dans l'espace public, ouvrant ainsi intellectuellement la voie à une appropriation large de ces travaux, questionnant le rôle social de l'historien dans la Cité. Les enjeux politiques de l'histoire coloniale ${ }^{29}$ ne cessent ainsi de scander dans l'espace public la question du postcolonialisme depuis 2005. Nécessairement, celle-ci n'est donc pas uniquement académique, accolant ainsi systématiquement à la figure du chercheur celle du militant. L'assomption des propositions historiographiques de l'ACHAC tient à ce moment politique où la colonisation est convoquée dans l'espace public ${ }^{30}$.

\section{Un dispositif éditorialement militant}

A rebours de la trajectoire de ce collectif, une stratégie éditoriale s'esquisse. A sa racine, il y a sans doute la crise de l'édition en sciences humaines. Parce qu'elle suppose la mise en concurrence des auteurs et des éditeurs, cette crise réclame un

27 L'essai de Jean-François Bayart peut être une introduction polémique aux débats, que balise la bibliographie de la fin du volume (Jean-François Bayart, Les études postcoloniales. Un carnaval académique, Paris, Khartala, coll. « Disputatio », 2010).

28 A titre d'exemple, pour Culture post-coloniale, 1961-2006, Paris, Autrement, 2006. Parmi les contributeurs : Robert Aldrich, professeur d'histoire à Sideney, Gabrielle Parker, titulaire d'une chaire à la School of Arts at Middlesex University, Françoise Vergès, politologue au Centre for Cultural Studies, Goldsmith Collège (Londres).

${ }^{29}$ Catherine Coquery-Vidrovitch, Les enjeux politiques del'histoire coloniale, Marseille, Agone, 2009.

30 Moment politique. L'ellipse désigne la forte politisation du postcolonial depuis 2005. Elle convie les discours sur les «émeutes » de novembre 2005, la politisation du fait colonial et ses suites dans la stratégie de Nicolas Sarkozy, illustrée notamment par le discours de Dakar (2007), le débat sur l'identité nationale (2009) et sa réfraction dans les droites, comme la genèse du mouvement des Indigènes de la République, ou l'écho du postcolonialisme dans la stratégie du Nouveau parti anticapitaliste (NPA)... 
Vincent Chambarlhac, «Fragments du jeu académique postcolonial (à propos d'un collectif, l'Association pour la connaissance de l'histoire de l'Afrique contemporaine, ACHAC) », Histoire@Politique. Politique, culture, société, $N^{\circ} 12$, septembre-décembre 2010, www.histoirepolitique.fr

savoir-faire spécifique dans le mode d’apparition de travaux qui concourent à la réputation d'un auteur. Il s'agit là de solliciter la polémique plus que la controverse scientifique. La première permet d'asseoir une réputation scientifique dans l'espace médiatique et, par contrecoup, de construire une place dans le dispositif institutionnel de la recherche (fondations, musées, chargés de recherche...). Le dispositif médiatique déployé joue sur deux registres noués par la figure du refoulé de la mémoire coloniale. Il reprend le « modèle » heuristique vichyssois, l'appliquant au postcolonialisme. Il s'agit de réaliser une «paxtonisation » du champ de l'historiographie du fait colonial en France selon Nicolas Bancel et Pascal Blanchard ${ }^{31}$. L'expression saisit la stratégie poursuivie et souligne un mode d'apparition dans l'espace public. Ce «modèle vichyssois » s'exprime en grande partie dans de courts articles confiés notamment au Monde diplomatique ${ }^{32}$. Ici, l'écriture, collective, se charge d'une évidence militante. L'essentiel est d'avancer l'argument quasi pathologique d'une amnésie, du refoulé d'une mémoire coloniale : le spectacle des zoos humains était ainsi routinier naguère, occulté aujourd'hui ${ }^{33}$. D'un syndrome l'autre, l'emprunt au lexique d'Henry Rousso est patent. Ces prises de position, qui sont le fait de chercheurs, s'entendent dans l'espace public comme un rapport singulier à l'histoire, construit sous les auspices du devoir de mémoire: animée par une écriture militante, la plume universitaire assoit la démonstration dans une revue de vulgarisation scientifique ${ }^{34}$. L'essentiel du dispositif éditorial tient à cette circulation incessante entre les pôles militant et heuristique.

La société française doit donc s'entrevoir et se questionner au prisme de l'héritage colonial. Amorcée dès 1997, cette logique culmine avec la fracture coloniale qui outrepasse l'essai d'histoire immédiate, posant les membres du collectif en praticiens et/ ou experts des pathologies sociales. L'ouvrage devient en 2010, sous la plume de Marie-Claude Smouts, le catalyseur des études postcoloniales sur la scène française $^{35}$ : le collectif fournit ainsi une première réponse à une demande issue du mouvement des Indigènes de la République comme des débats autour de l'abrogation de l'article 4 de la loi du 23 février 2005. Les émeutes de novembre 2005 renchérissent médiatiquement l'hypothèse d'une pertinence de la grille postcoloniale comme lecture du social. Un effet de seuil a été franchi. Dans ce franchissement, l'évidence d'une situation postcoloniale s'est imposée. La logique du palimpseste, inhérente à l'intrication des enjeux dans la polémique sur la question coloniale,

\footnotetext{
31 Nicolas Bancel, Pascal Blanchard, «Les pièges de la mémoire coloniale », Les Cahiers français. La mémoire, entre histoire et politique, juillet-août 2001, n 303.

32 Nicolas Bancel et alii, « Une histoire coloniale refoulée », Le Monde diplomatique, juin 2001; Nicolas Bancel et alii, « Le miroir colonial brisé », Le Monde diplomatique, juin 2001.

${ }_{33}$ Nicolas Bancel et alii, « Le spectacle routinier des zoos humains », Le Monde Diplomatique « Manière de voir » $n^{\circ} 58$, juillet-août 2001.

34 Nicolas Bancel, Pascal Blanchard , «Les pièges de la mémoire coloniale », Les Cahiers français. La mémoire, entre histoire et politique, juillet-août 2001, n 303. Nicolas Bancel et alii, « Les non-dits de l'antiracisme français : la République coloniale », dans « Politique et altérité : la société française face au racisme », Cahiers de la Méditerranée, $n^{\circ}$ 60, décembre 2001

35 Marie-Claude Smouts, «Les études postcoloniales en France: émergence et résistances », dans Nicolas Bancel et alii, Ruptures postcoloniales. Les nouveaux visages de la société française, Paris, La Découverte, 2010, p. 312.
} 
Vincent Chambarlhac, «Fragments du jeu académique postcolonial (à propos d'un collectif, l'Association pour la connaissance de l'histoire de l'Afrique contemporaine, ACHAC) », Histoire@Politique. Politique, culture, société, $N^{\circ} 12$, septembre-décembre 2010, www.histoirepolitique.fr

recouvre alors la part de la structuration intellectuelle du débat. Formulée dans l'après-coup (2010), l'analyse de Marie-Claude Smouts mesure l'effacement de la stratégie éditoriale au moment où celle-ci trouve dans l'espace public l'écho qui construit le collectif en praticien du social au nom du postcolonialisme. En son moment politique, la trajectoire de l'ACHAC consacre l'irruption médiatique du postcolonialisme dans le champ scientifique. Un «tournant postcolonial » serait là à l'œuvre dont l'ouvrage Ruptures postcoloniales entend sonder les contours, tout en gardant à « l'esprit l'illégitimité universitaire de l'histoire postcoloniale » en 2010. Puisque l'engagement du collectif peut se «concilier avec des approches plus distanciées d'une construction de l'objet respectant une certaine façon de se rapporter aux événements, aux autres et à soi-même ${ }^{36}$ », la capacité de ces historiens à diagnostiquer les pathologies sociales certifie leur scientificité. Dans cette configuration tautologique, l'historien du postcolonialisme est scientifique car intrinsèquement militant. La boucle est bouclée, la reconnaissance scientifique procède de la capacité militante à distancier son engagement pour construire un objet, posé ensuite comme central dans le réagencement du champ académique. L'illégitimité universitaire du postcolonialisme procède d'un combat d'arrière-garde. Coda, donc?

\section{S'inscrire en challenger}

Ce dispositif éditorial se singularise par sa dynamique réflexive entre des pôles discursifs, schématiquement qualifiés de militant (Le Monde Diplomatique, les pages « Débats » du Monde, les interventions radiophoniques chez Daniel Mermet...), d'heuristique (des revues de haut niveau de vulgarisation comme les Cahiers français, Hommes et migrations...) et les thématiques développées dans l'espace public par les politiques de mémoire et les luttes pour la reconnaissance ${ }^{37}$. C'est dans cette réflexivité que se saisit la part du jeu à l'œuvre, par quoi des challengers tentent de s'inscrire dans le champ scientifique, certes au prix de frottements. Lire cette stratégie en termes d'inscriptions académiques implique à nouveau la périodisation ; des figures et des rhétoriques s'enchâssent qui représentent autant de modalités d'être académiquement à ce « concept circulant »- selon la formule de l'introduction de Ruptures postcoloniales - qu'est le postcolonialisme.

Née du combat pour les droits civiques aux Etats-Unis, la discrimination positive permit le développement de la Public history ${ }^{38}$. La naissance, puis le développement

\footnotetext{
36 L'ensemble des citations provient de l'introduction collective à Ruptures postcoloniales. Cf. « De la fracture coloniale aux ruptures postcoloniales », dans Nicolas Bancel et alii, Ruptures postcoloniales, op. cit., p. 15, 29.

37 Marilyne Crivello, Patrick Garcia, Nicolas Offenstadt (dir.), Concurrences des passés. Usages politiques du passé dans la France contemporaine, Marseille, PUP, 2006.

38 Henry Rousso, "L'histoire appliquée ou les historiens thaumaturges », Vingtième siècle. Revue d'histoire, 1984, n 1 . Olivier Dumoulin, Le rôle social del'historien, Paris, Albin Michel, 2002.
} 
Vincent Chambarlhac, «Fragments du jeu académique postcolonial (à propos d'un collectif, l'Association pour la connaissance de l'histoire de l'Afrique contemporaine, ACHAC) », Histoire@Politique. Politique, culture, société, $N^{\circ} 12$, septembre-décembre 2010, www.histoirepolitique.fr

de l'agence des Bâtisseurs de mémoire, comme celui de l'ACHAC, procèdent sans doute de ce lien. La charge critique de l'article de Camille Trabendi pour Agone sur Pascal Blanchard, free lance researcher, s'entend dans cette configuration ${ }^{39}$. La polémique qui s'ensuit sur le blog d'Agone ${ }^{40}$ porte comme titre le néologisme de Postcolonial-business; l'article indique qu'il y avait dans «l'interprétation économique et sociale du malaise des classes populaires » comme un « marché à conquérir » pour Pascal Blanchard. Articulée sur la trajectoire de l'ACHAC en son moment politique, la charge déconstruit la posture de l'historien; elle dit peu sur l'inscription de ce Postcolonial-business dans le champ académique, sinon par le rappel de l'appartenance au laboratoire GDR 2322 du CNRS lue comme « une amulette qui préserve des pairs malveillants ${ }^{41}$ ». L'argument du marché académique revient également sous la plume de J ean-François Bayart, analysant la « soudaine promotion des postcolonial studies » comme « une stratégie de la niche de la part de chercheurs en quête d'une part du marché académique ${ }^{42}$ ». Polémiques, circonstanciées ou fouillées, ces analyses témoignent de l'irruption du postcolonial dans le champ académique. La critique énoncée sous les auspices d'une logique des places dit finalement peu du postcolonialisme, mais beaucoup dans le cas de l'ACHAC du rapport de cette dernière au champ académique, lui-même en crise. Elle insiste peu sur l'art de faire de l'ACHAC par quoi ses problématiques s'arriment à l'histoire scientifique nationale. Sa production éditoriale éclaire celui-ci.

A des livres ou des articles inscrits dans le présent de la situation française symbolisés par La Fracture coloniale (2005) ou Ruptures postcoloniales (2010) marqués par le tempo du chercheur militant, l'ACHAC associe une production davantage centrée sur le questionnement d'un objet historique : la culture coloniale pour le triptyque des éditions Autrement (2003-2006) et les guerres de mémoires à La Découverte en $2008^{43}$. Ce dernier ouvrage importe. Son introduction rappelle l'article fondateur de Daniel Lindenberg pour la revue Vingtième siècle. Revue d'histoire, "Guerres de mémoire en France 44 ». Publié en 1994, ce dossier tisse un lien entre les guerres de mémoires telles qu'elles s'entrevoient alors, et la notion de guerre franco-française avancée une décennie plus tôt par la même revue. Le postcolonialisme de l'ACHAC s'introduit explicitement dans une lecture classique du fait républicain, puisque la culture coloniale s'arrime, par l'introduction, aux guerres franco-françaises, devenues de mémoires. La translation conceptuelle mérite que l'on

\footnotetext{
39 Il s'agit d'un pseudonyme. Camille Trabendi, « Pascal Blanchard en "free lance researcher" », Agone $n^{\circ}$ 41-42 (« Les intellectuels, la critique et le pouvoir »), 2009, p. 185-192.

40 Pour Agone, voir la réponse de Sylvain Laurens ( « Les vertus curatives de la boue : lettre ouverte à Pascal Blanchard et pes 18 mai 2010, http:// blog.agone.org/post/2010/05/18/Postcolonial-Business-1). Pascal Blanchard s'en prend $\begin{array}{lllll}\text { violemment } & \text { à } & \text { Camille } & \text { Trabendi } & \text { sur }\end{array}$ http://sites.radiofrance.fr/ franceinter/ em/ ete/ cavousderange/

${ }^{41}$ Camille Trabendi, « Pascal Blanchard en "free lance researcher" », art. cité, p. 187.

42 J ean-François Bayart, Les études postcoloniales, op. cit., p. 38.

43 Pascal Blanchard, Isabelle Veyrat-Masson (dir.), Les guerres de mémoires. La France et son histoire, Paris, La Découverte, 2008.

44 Daniel Lindenberg, « Guerres de mémoire en France », Vingtième siècle. Revue d'histoire, n 44,avriljuin 1994.
} 
Vincent Chambarlhac, «Fragments du jeu académique postcolonial (à propos d'un collectif, l'Association pour la connaissance de l'histoire de l'Afrique contemporaine, ACHAC) », Histoire@Politique. Politique, culture, société, $N^{\circ} 12$, septembre-décembre 2010, www.histoirepolitique.fr

s'y attarde. Elle dessine une stratégie épistémologique où des thématiques développées au sein de secteurs réputés marginaux s'ancrent dans l'histoire politique. L'histoire coloniale, l'histoire des médias, l'approche en sciences de l'éducation, s'unissent dans l'introduction pour promouvoir un objet qui interroge l'histoire politique et se propose de reconceptualiser l'une de ses grilles de lecture au titre d'un nouvel état de la discipline. Dans ce jeu, la culture coloniale, naguère donnée comme une pratique réfractée des colonial studies soulignant le retard d'une part de l'historiographie française, s'efface devant l'argument des guerres de mémoires, concept valise apte à lier des dispositifs auparavant irréductibles l'un à l'autre. L'argument englobant le colonial des guerres de mémoires fait ainsi apparaître une grammaire de leurs formes, de leurs thèmes. Si l'introduction n'entend pas établir un nouveau paradigme, la dimension collective du livre doit se lire, selon elle, comme l'expression d'une somme et d'une mise au point au centre du - supposé - nouveau dispositif historiographique. Reste que cette centralité s'entend dans l'unique rapport du postcolonialisme décliné par l'ACHAC au fait républicain « présentifié » et, pour une grande part, déshistoricisé : lue dans l'horizon structurel des guerres francofrançaises, les guerres de mémoires - en tant qu'elles convoquent de manière conflictuelle des mémoires coloniales - se révèlent comme un énième avatar des fièvres hexagonales. $\mathrm{Ni}$ plus, ni moins. Tel est le prix historiographique de l'opération : l'oubli de la singularité des propositions des postcolonial studies, qui ne bousculent plus l'édifice du modèle républicain, mais s'agrègent à ce dernier. Le monument historiographique des travaux sur la République dicte aux challengers les conditions de sa visite.

\section{Conclure?}

Questionner le postcolonialisme à la lumière de la trajectoire intellectuelle de l'ACHAC dans son rapport à l'histoire dit peu, finalement, des postcolonial studies elles-mêmes - l'analyse de la politique de traduction déployée par les éditions Amsterdam serait à cet égard plus éclairante. La bibliographie de l'essai de J eanFrançois Bayart compte deux cent quarante titres, trois seulement sont de Nicolas Bancel et Pascal Blanchard, classés à la rubrique « La Bibliothèque coloniale, pour le meilleur et pour le pire...» : soit un article («Qui a peur du postcolonial ? ») et deux ouvrages La Fracture coloniale et La République coloniale ${ }^{45}$. Les titres valent indices, ils circonscrivent la stratégie poursuivie par l'ACHAC entre science et militantisme, impliquent que le seul rapport au fait républicain fait tenir étroitement

\footnotetext{
45 J ean-François Bayart, Les études postcoloniales, op. cit., p. 99-100. Les références exactes sont: Nicolas Bancel, Pascal Blanchard, Paul Gilroy, Achille Mbembre, «Qui a peur du postcolonial ? », Mouvements, $n^{\circ}$ 51, 2007. Et Nicolas Bancel, Pascal Blanchard, Sandrine Lemaire (dir.), La Fracture coloniale. La société française au prisme de l'héritage colonial, Paris, La Découverte, 2005. Nicolas Bancel, Pascal Blanchard, Françoise Vergès, La République coloniale. Essai sur une utopie, Paris, Albin Michel, 2003.
} 
Vincent Chambarlhac, «Fragments du jeu académique postcolonial (à propos d'un collectif, l'Association pour la connaissance de l'histoire de l'Afrique contemporaine, ACHAC) », Histoire@Politique. Politique, culture, société, $N^{\circ} 12$, septembre-décembre 2010, www.histoirepolitique.fr

ces figures. En somme, cette trajectoire se tient en lisière des controverses académiques sur le postcolonialisme et s'effectue davantage dans un jeu sur l'historiographie politique classique. C'est là le prix d'un art de faire éditorial et académique que je me suis efforcé de décrire.

Pour autant, si elle est patente, la logique des places échoue à restituer seule le sel de cette trajectoire entre médiatisation, politique et travail historique. Le collectif de l'ACHAC dans son rapport à l'histoire joue sans doute le rôle d'une butte-témoin. Il est indice d'une séquence historiographique complexe où les questions de l'édition en sciences humaines et sociales se mêlent à l'ouverture de nouveaux fronts historiographiques. Il est signe d'une approche « présentiste » du passé dont l'un des usages publics réclame que la recherche arme les revendications dans l'horizon des politiques de reconnaissance. La scientificité de l'historien est là immédiatement militante - à son corps défendant, ou, comme ici, assumée comme telle. Il est trace, enfin, d'un jeu toujours mouvant des challengers historiographiques sur l'un des môles de l'historiographie politique française, le fait républicain. Qu'à cet égard l'appel à une « paxtonisation » des études coloniales vaille feuille de route dès 2001 prouve qu'en ce domaine, la rupture épistémologique se conçoit avec une part des outils qui firent l'histoire du temps présent. Faut-il employer le terme de rupture?

\section{L'auteur \\ Les travaux de Vincent Chambarlhac, maître de conférences, portent principalement sur l'épistémologie, le rapport des intellectuels au XX ${ }^{\mathrm{e}}$ siècle au mouvement ouvrier et social au sein du centre Georges Chevrier (UMR 5605). Vincent Chambarlhac est membre du comité de rédaction de Dissidences, des Cahiers d'histoire, revue d'histoire critique. \\ Résumé \\ La trajectoire historiographique de l'l'Association pour la connaissance de l'histoire de l'Afrique contemporaine (ACHAC) repère la manière dont l'un des segments du postcolonialisme s'amarre au champ scientifique. La stratégie poursuivie par ce collectif se déploie sur deux registres, militants, scientifiques. A l'intersection de ces registres, le postcolonialisme s'ancre dans une séquence actuelle marquée par sa forte politisation.}

\section{Résumé}

La trajectoire historiographique de l'Association pour la connaissance de l'histoire de l'Afrique contemporaine (ACHAC) repère la manière dont l'un des segments du postcolonialisme s'amarre au champ scientifique. La stratégie poursuivie par ce collectif se déploie sur deux registres, militants, scientifiques. A l'intersection de ces registres, le postcolonialisme s'ancre dans une séquence actuelle marquée par sa forte politisation.

\footnotetext{
Abstract

Fragments of the postcolonial academic game (about a collective : l'Association pour la connaissance de l'histoire de l'Afrique contemporaine - ACHAC)

The historiography trajectory of ACHAC points out how one of the post colonialism segment is connected to the scientific field. The strategy that this group is following is
} 
Vincent Chambarlhac, «Fragments du jeu académique postcolonial (à propos d'un collectif, l'Association pour la connaissance de l'histoire de l'Afrique contemporaine, ACHAC) », Histoire@Politique. Politique, culture, société, N¹2, septembre-décembre 2010, www.histoirepolitique.fr

spreading over two registries: activist and scientific. The post colonialism in a topical sequence marked by its strong politicization where these two registries meet.

Mots clés : postcolonialisme; France; historiographie; édition; intellectuels ; politique.

Key words : postcolonialism; France; historiography; publishing ; intellectuals; politics.

Pour citer cet article : Vincent Chambarlhac, « Fragments du jeu académique postcolonial (à propos d'un collectif, l'Association pour la connaissance de l'histoire de l'Afrique contemporaine, ACHAC) », Histoire@Politique. Politique, culture, société, $\mathrm{N}^{\circ} 12$, septembre-décembre 2010, www.histoire-politique.fr 